Proceedings of the 35th European Peptide Symposium Patrick B. Timmons, Chandralal M. Hewage, Michal Lebl (Editors)

\title{
Novel peptide-based control measures against the rice fungal pathogen Pyricularia oryzae
}

Marta De Zotti ${ }^{1}$, Luca Sella ${ }^{1}$, A. Quarantin ${ }^{1}$, Carla Castiglioni ${ }^{1}$, Angela Bolzonello ${ }^{1}$, Francesco Favaron ${ }^{1}$, A.M. Picco ${ }^{2}$, R. Govind ${ }^{1}$, Silvio Tundo ${ }^{1}$, Silv ana Odorizzi ${ }^{1}$, Le T. Do ${ }^{3}$, Vu V. Van ${ }^{4}$, Nguyen M. Hung ${ }^{3}$

${ }^{1}$ University of Padova, Italy

${ }^{2}$ University of Pavia, Italy

${ }^{3}$ Duy Tan University, Viet Nam

${ }^{4}$ Nguyen Tat Thanh University, Viet Nam

https://doi.org/10.17952/35EPS.2018.248

\section{Introduction}

The filamentous fungus Pyricularia oryzae is the main causal agent of the rice blast disease, which accounts for $10-30 \%$ yield losses per year globally (Talbot, 2003). The disease is worldwide distributed in more than 85 countries, including Italy and Vietnam (Fig. 1; Talbot, 2003), and causes typical leaf spot eye shaped symptoms characterized by large, spreading lesions with a necrotic center and a chlorotic margin (Fig. 1; Wilson and Talbot, 2009).
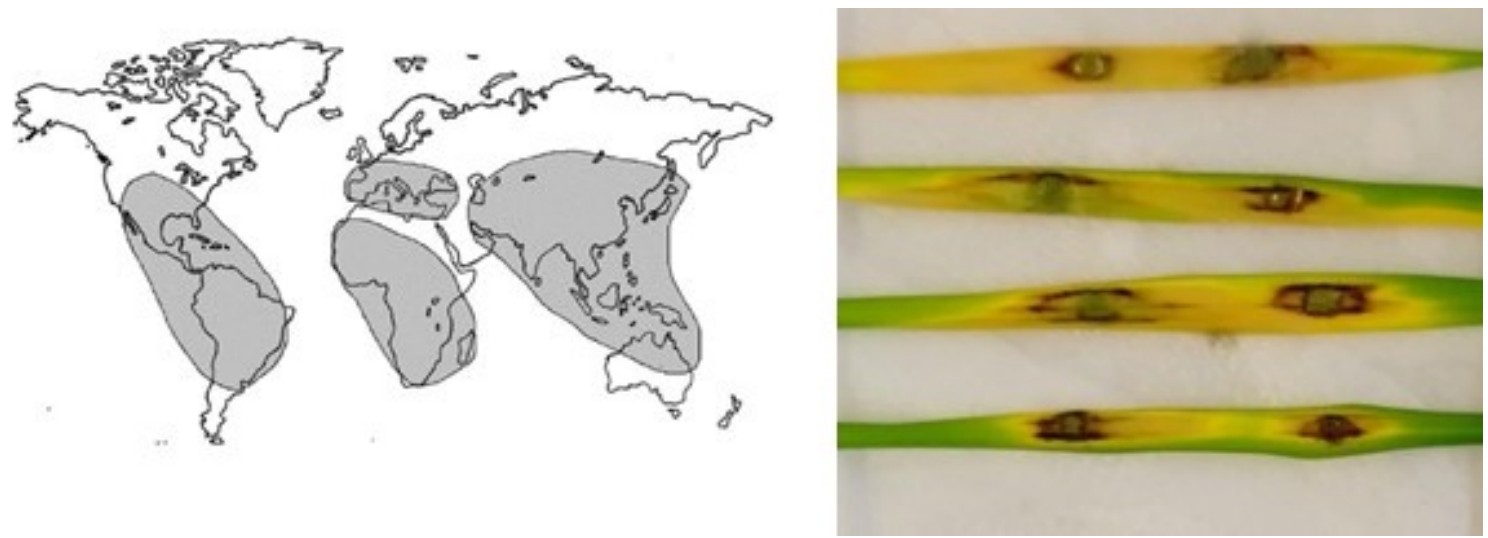

Figure 1: Left: global distribution of rice blast disease. Right: typical blast disease symptoms on rice leaves.

P. oryzae has been recently ranked as the most important fungal pathogen of crops (Fig. 2; Dean et al., 2012).

\begin{tabular}{ll}
\hline Rank & Fungal pathogen \\
\hline 1 & Magnaporthe oryzae \\
2 & Botrytis cinerea \\
3 & Puccinia spp. \\
4 & Fusarium graminearum \\
5 & Fusarium oxysporum
\end{tabular}

Figure 2: Most important fungal pathogens of crops worldwide.

New antimicrobial peptides, analogs of the natural peptaibol trichogin GA IV (tric), have been synthesized (De Zotti et al., 2009; De Zotti et al., 2012) and tested in vitro against several $P$. oryzae strains from different geographic origin. Trichogin GA IV is the main component of the mixture of peptide congeners produced by Trichoderma longibrachiatum as part of its defense mechanism against other microorganisms (Battaglia et al., 2013). As all peptaibols, its sequence is characterized by the presence of the non-coded residue Aib ( $\alpha$ aminoisobutyric acid), a C-terminal 1,2-aminoalcohol (Leucinol, Lol) and an acylated N-terminus(1-Octanoyl). All peptide sequences are reported in Table 1. 
Table 1: Primary structure of tested trichogin analogs.

\begin{tabular}{|l|l|}
\hline Abbreviation & Sequence \\
\hline tric & 1-octanoyl-Aib-Gly-Leu-Aib-Gly-Gly-Leu-Aib-Gly-Ile-Lol \\
\hline K2 & 1-octanoyl-Aib-Lys-Leu-Aib-Gly-Gly-Leu-Aib-Gly-Ile-Lol \\
\hline K259G6 & 1-octanoyl-Aib-Lys-Leu-Aib-Lys-Gly-Leu-Aib-Lys-Ile-Lol \\
\hline K56 & 1-octanoyl-Aib-Gly-Leu-Aib-Lys-Lys-Leu-Aib-Gly-Ile-Lol \\
\hline K5 & 1-octanoyl-Aib-Gly-Leu-Aib-Lys-Gly-Leu-Aib-Gly-Ile-Lol \\
\hline K6 & 1-octanoyl-Aib-Gly-Leu-Aib-Gly-Lys-Leu-Aib-Gly-Ile-Lol \\
\hline
\end{tabular}

\section{Results \& Discussion}

\section{Synthesis}

All analogs were synthesized by SPPS using a cost-effective strategy with Oxyma Pure / DIC (diisopropylcarbodiimide) as activating agents. All peptides were obtained in high yield and crude purities.

Conformational analysis

Several spectroscopic techniques were applied to shed light on the 3D-structure preferably adopted by the synthesized analogs in solution. Our Circular Dichroism (CD) analysis showed that all peptides mainly assume a right-handed310-helical conformation in a membrane-mimicking environment(Fig. 3).

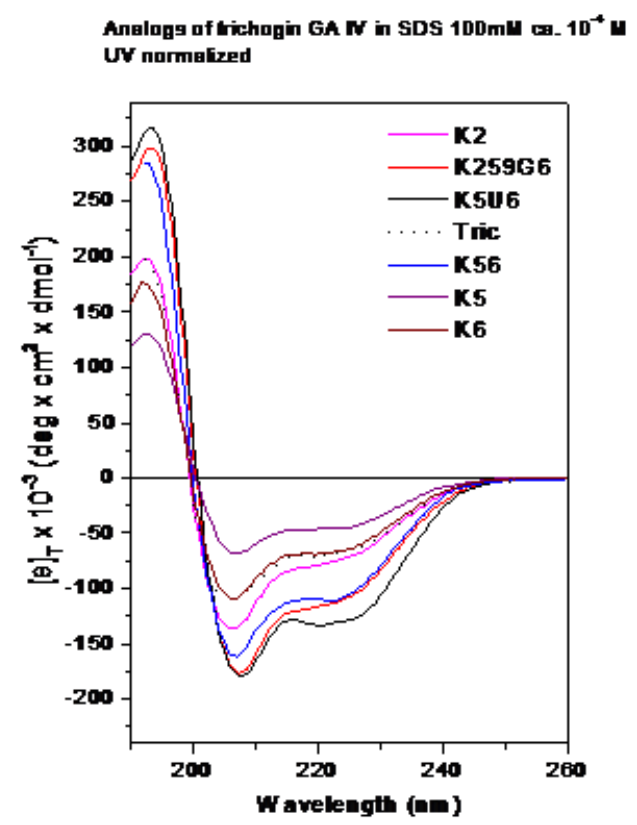

Figure 3: CD spectra of selected analogs in aqueous SDS 100mM (peptide concentration: $0.1 \mathrm{mM}$ ).

\section{Antifungal activity}

The synthesized peptides have been screened in vitro against two different strains (AP and Guy11) of the rice fungal pathogen $P$. oryzae. In vitro germination and growth tests conducted in microtiter plates inoculating conidia in potato dextrose medium in presence of peptides showed significant differences in the biocidal activity among the synthesized peptaibols. Although the assay revealed some differences in efficacy between strains, all analogs showed a strong inhibitory effect against $P$. oryzae at $50 \mu \mathrm{M}$ concentration.

\section{Microscopy}

The effect of the most active peptides on fungal conidia was also verified by optical microscopy analysis. In particular, the most effective peptides resulted able to inhibit spore germination and caused in fungal cells morphological modifications probably involving vacuolation and membrane damages. 


\section{Conclusions}

We exploited a cost-effective, solid-phase synthetic strategy to afford trichogin GA IV analogs with high yield and purity. The conformational studies showed that the analogs adopt a 310-helical structure regardless the sequence modifications. Preliminary tests have highlighted that these peptaibol analogs are non-toxic against plant cells.

Our in vitro screening and microscopic analysis have allowed to identify several peptide sequences very effective in inhibiting $P$. oryzae spore germination and fungal growth. The most effective sequences are currently being tested in vivo on rice leaves to verify their possible efficacy in protecting rice from the blast disease.

\section{References}

Battaglia D. et al., Mol. Plant-Microbe Interact. 2013, 26: 1249. DOI: 10.1094/MPMI-02-13-0059-R

Dean R. et al., Mol. Plant Pathol. 2012, 13: 414-430. DOI: 10.1111/j.1364-3703.2011.00783.x

De Zotti M. et al., J. Pept. Sci. 2009, 15: 615. DOI: 10.1002/psc.1135

De Zotti M. et al., Amino Acids, 2012, 43: 1761. DOI: 10.1007/s00726-012-1261-7

Talbot N.J., Annu. Rev. Microbiol. 2003, 57: 177-202. DOI: 10.1146/annurev.micro.57.030502

Wilson R.A. and Talbot N.J., 2009, Nature Rev. Microbiol. 2009, 7: 185-195. DOI: 10.1038/nrmicro2032

\section{Acknowledgments}

This work is part of the Scientific and Technological Cooperation Agreement between the Italian Ministry of Foreign Affairs and International Cooperation and the Departmentof International Cooperation of the Ministry of Science and Technology of Vietnam. 